\title{
Criminologie
}

\section{« Faire son temps » et « attendre »}

Temporalités carcérales et temps vécu dedans et dehors

"Doing time" and "waiting"

Carceral rhythms and the experience of time inside and

outside prison

\section{“Cumplir su pena” y "esperar" \\ Temporalidades carcelarias y el tiempo vivido adentro y afuera}

\section{Gwenola Ricordeau}

Volume 52, numéro 1, printemps 2019

Les proches de personnes judiciarisées : expériences humaines et connaissances carcérales

URI : https://id.erudit.org/iderudit/1059539ar

DOI : https://doi.org/10.7202/1059539ar

Aller au sommaire du numéro

\section{Éditeur(s)}

Les Presses de l’Université de Montréal

ISSN

0316-0041 (imprimé)

1492-1367 (numérique)

Découvrir la revue

Citer cet article

Ricordeau, G. (2019). « Faire son temps » et « attendre » : temporalités carcérales et temps vécu dedans et dehors. Criminologie, 52(1), 57-72. https://doi.org/10.7202/1059539ar

\section{Résumé de l'article}

Basé sur une recherche de terrain menée en France auprès de personnes détenues et de proches de prisonniers, cet article interroge les diverses manières dont le temps est vécu dedans et dehors au cours des différentes phases de l'incarcération et comment celles-ci sont mises en présence d'autres rythmes sociaux, en particulier l'injonction qui est faite aux prisonniers de " donner du sens à leur peine » (ou « faire son temps »), alors que les proches sont assignés à une position d'attente. L'auteure décrit d'abord les différentes expériences du temps (dedans et dehors), qui varient selon la durée de la peine et le temps déjà effectué. L'auteure décrit ensuite diverses formes de discordance entre le temps vécu dedans et dehors et la manière dont elles se traduisent dans les relations et solidarités familiales. Enfin, l'auteure analyse comment les prisonniers, les anciens prisonniers et leurs proches rationalisent un temps initialement pensé comme " perdu » et comment leurs stratégies sont influencées par la longueur de la peine et le type de prison où la peine a été effectuée.
Tous droits réservés @ Les Presses de l’Université de Montréal, 2019
Ce document est protégé par la loi sur le droit d'auteur. L’utilisation des services d'Érudit (y compris la reproduction) est assujettie à sa politique d'utilisation que vous pouvez consulter en ligne.

https://apropos.erudit.org/fr/usagers/politique-dutilisation/ 


\title{
« Faire son temps » et « attendre » \\ Temporalités carcérales et temps vécu dedans et dehors
}

\author{
Gwenola Ricordeau ${ }^{1}$ \\ Professeure assistante, justice criminelle \\ Université d'État de Californie, Chico \\ gricordeau@csuchico.edu
}

RÉSUMÉ - Basé sur une recherche de terrain menée en France auprès de personnes détenues et de proches de prisonniers, cet article interroge les diverses manières dont le temps est vécu dedans et dehors au cours des différentes phases de l'incarcération et comment celles-ci sont mises en présence d'autres rythmes sociaux, en particulier l'injonction qui est faite aux prisonniers de "donner du sens à leur peine» (ou "faire son temps»), alors que les proches sont assignés à une position d'attente. L'auteure décrit d'abord les différentes expériences du temps (dedans et dehors), qui varient selon la durée de la peine et le temps déjà effectué. L'auteure décrit ensuite diverses formes de discordance entre le temps vécu dedans et dehors et la manière dont elles se traduisent dans les relations et solidarités familiales. Enfin, l'auteure analyse comment les prisonniers, les anciens prisonniers et leurs proches rationalisent un temps initialement pensé comme "perdu» et comment leurs stratégies sont influencées par la longueur de la peine et le type de prison où la peine a été effectuée.

MotS CLÉs - Prison, détenus, détenues, visite, temps, proches.

\section{Introduction}

Sous ses différentes acceptions, le temps a été abondamment exploré par les travaux sur la prison, notamment à travers la question de la socialisation carcérale (ou prisonization). Depuis le travail pionnier de Clemmer (1940), les effets du temps passé en prison sur les personnes détenues constituent même un sujet classique du champ. Il a été abon-

1. Department of Political Science and Criminal Justice, California State University, Chico, Butte Hall, 400 W First Street, Chico, Californie, États-Unis, 95929-0455.

Criminologie, vol. 52, $\mathrm{n}^{\circ} 1$ (2019) 
damment discuté (Adams, 1992; Garabedian, 1963; Montandon et Crettaz, 1981, p. 177-178; Moran, 2012; Wheeler, 1961, p. 706), y compris dans le cadre plus large des «institutions totales» (Goffman, 1968, p. 104-111). Des travaux de type ethnographique menés en détention (voir en particulier Cunha, 1997; Leal et Mond, 2001; Marchetti, 2001; Middlemass et Smiley, 2016; Pauchet, 1984) ont permis de renouveler ces questionnements. Les approches biographiques, notamment en termes de trajectoires carcérales (Chantraine, 2003), en transformant l'appréhension de l'établissement carcéral, ont naturellement placé la question des temporalités au centre du champ. Ces travaux ignorent, pour l'essentiel, les proches des personnes détenues. Simultanément, les recherches sur les proches de détenus, malgré leur développement (voir notamment Ricordeau, 2008; Touraut, 2012), en particulier en Amérique du Nord (par ex.: Braman, 2007; Comfort, 2008), évoquent fréquemment qu'ils doivent faire face aux temporalités carcérales, mais cet angle est rarement celui que privilégient ces analyses (voir néanmoins Muth et Walker, 2013).

Les articulations entre les temps individuels et les temps institutionnels (Pauchet, 1984), notamment à travers l'injonction constamment faite aux personnes incarcérées de «donner du sens» à la peine, c'està-dire au temps, ont été abondamment explorées. En rendant compte, dans cet article, des manières dont, sous l'angle des solidarités conjugales et familiales, les rythmes carcéraux et les différents temps de la peine se heurtent à ceux du dehors, je déplace donc une focale ordinairement mise sur la détention - où il y a certes beaucoup à dire sur les tensions entre différentes temporalités (celles des détenus, mais également entre celles des détenus et des membres du personnel). Il s'agit ici plutôt, à partir des solidarités conjugales et familiales, d'explorer les diverses manières dont le temps est vécu par les prisonniers et par leurs proches, et comment cette expérience du temps carcéral est façonnée par les rythmes judiciaires et institutionnels. Il s'agit par ailleurs d'interroger les formes de discordance entre le temps vécu dedans et dehors (par exemple au moment des visites) et d'explorer comment le fait de se trouver devant différents usages du temps peut se traduire par l'effritement des solidarités conjugales et familiales.

Les réflexions exposées ici sont tirées de mes recherches, menées depuis une dizaine d'années, sur le système carcéral français, principalement sous l'angle des relations entre les personnes détenues et leurs proches (Ricordeau, 2008). Outre une expérience personnelle 
de l'incarcération de proches qui donne une dimension autoethnographique à mes travaux, s'ajoute une enquête de terrain qui repose notamment sur la réalisation en 2003 d'entretiens avec plus de 25 proches de détenus (essentiellement des compagnes et des mères) et près de 80 personnes incarcérées dans cinq prisons: deux maisons d'arrêt, deux centres de détention et une maison centrale. Les personnes interviewées en prison sont très majoritairement des hommes condamnés. Néanmoins, cet article évoque aussi les personnes prévenues, qui constituent une part importante des personnes incarcérées en France ${ }^{2}$. Comme dans toute enquête en prison, la question du temps s'est invitée: d'abord, car la sociologue peut découvrir, avec l'épreuve du terrain, son instrumentalisation pour «occuper» les détenus et que ceux-ci sont loin d'«avoir tout leur temps» (voir plus loin); ensuite, car les interviewés abordent souvent spontanément le «temps»-celui de la détention ou celui que «prend» la prison aux personnes dont un proche est incarcéré.

J'examine d'abord l'expérience du temps vécu par les personnes incarcérées, notamment selon le temps déjà passé en détention et la durée des peines prononcées ou encourues. J'explore ensuite la confrontation du temps vécu par les personnes détenues (en particulier au début et en fin de la peine) avec les discordances entre les rythmes du dedans et du dehors. Enfin, je décris les stratégies déployées par les personnes détenues et les sortants de prison afin de rationaliser un temps a priori considéré comme «perdu».

\section{Des peines et du temps}

À l'écoute des personnes détenues, apparaît d'abord l'unicité de l'expérience du temps en prison: elle est réductible à un temps qu'il faut «passer» ou «faire passer». Pourtant, selon les caractéristiques individuelles, mais aussi les établissements d'affectation et les régimes de détention auxquels les personnes sont soumises, s'esquissent des rythmes propres à la vie en détention et des phases au cours des trajectoires carcérales qui ne sont pas étrangères aux manières dont la solidarité familiale s'organise lorsqu'une personne est incarcérée.

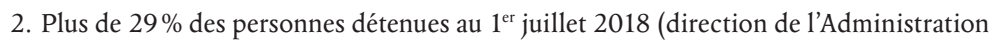
pénitentiaire, 2018). 


\section{Les temps de la prison}

Les recherches sur les temporalités des peines de prison ont été nombreuses depuis le travail de Clemmer (1940). Sa typologie des différentes phases de la socialisation carcérale a été particulièrement féconde (notamment Goffman, 1968, p. 104-111; Montandon et Crettaz, 1981, p. 177-178). Malgré les discussions qu'elle a suscitées - en particulier l'accent mis par Irwin et Cressey (1962, p. 142-155) sur l'influence des attitudes et des valeurs possédées par les individus avant leur incarcération -, elle permet de souligner la diversité des expériences du temps par les prisonniers.

L'expérience du temps carcéral varie selon que la personne est prévenue ou condamnée, selon le temps déjà passé en détention ou la durée de la peine déjà effectuée et celle qui reste à purger, selon que la personne condamnée dispose d'une date de sortie ou non (réclusion criminelle à perpétuité) et selon le type d'établissement d'affectation. Mais le temps vécu dépend aussi du sexe ${ }^{3}$ (en particulier du rythme différentiel de la procréation chez les hommes et chez les femmes), de l'âge et des ressources individuelles (notamment intellectuelles).

Même si la diversité des manières de «faire son temps» rend difficile toute forme de généralisation, il ressort que l'arrivée en détention et l'attente du procès (la détention préventive) sont marquées par l'incertitude - bien loin des formes de routinisation qui s'observent chez les détenus purgeant les peines les plus longues dans les établissements pour peine. Par ailleurs, le temps en prison est structurellement projeté et l'objet de pronostics ${ }^{4}$. Enfin, l'incarcération s'accompagne de divers apprentissages, à commencer par les manières de "passer le temps» (voir plus loin). Mais ces temps de la peine s'inscrivent aussi dans des temporalités extérieures que façonnent les solidarités conjugales et familiales.

\section{Les temps des solidarités conjugales et familiales}

Le début de l'incarcération est généralement décrit comme le moment le plus douloureux de l'expérience de la prison, concentrant la plupart des ruptures et des déceptions (« Tu tombes de haut... Tout le monde

3. En France, un proverbe de prison affirme qu'«un an pour une femme, c'est comme deux ans pour un homme».

4. Il est remarquable que même aux détenus se déclarant innocents, il soit demandé «combien?». 
te lâche...»). Il constitue, pour les solidarités familiales, un «moment de vérité», car les ruptures se produisent proportionnellement davantage durant cette période (Cassan, Toulemon et Kensey, 2002, p. 43). Mais le début de l'incarcération peut aussi être l'occasion de renouer ou de raviver des liens distendus. La plupart des détenus évoquent leur envie frénétique d'écrire à leurs proches pendant les premiers temps de l'incarcération. La correspondance, souvent intensive au début et qui ponctue la vie en détention (l'arrivée du courrier, en semaine), tend à diminuer par la suite (Fabiani et Soldini, 1995, p. 261).

L'importance prise par les relations conjugales et familiales au début de l'incarcération est renforcée par les interventions sociales qui les mettent souvent à contribution. Ces interventions se concentrent, au cours de la détention, à trois moments, parfois concomitants pour les deux premiers: à l'arrivée en détention, avant la condamnation (enquête sociale, de moralité, etc.) et à l'approche de la sortie. Le début de la détention est marqué par l'urgence, car les démarches à effectuer sont nombreuses. Il est suivi par le «temps mort» de l'intervention sociale qu'est le déroulement de la peine jusqu'à la survenue des démarches liées à la demande de libération ou à la sortie elle-même (recherche de logement, d'emploi, etc.).

$\mathrm{Au}$ cours de leur incarcération, justement dans ce «temps mort» de l'intervention sociale, beaucoup de détenus sont de moins en moins soutenus, notamment financièrement, et ce qui était naturel au début de l'incarcération devient exceptionnel et doit être sollicité. Par ailleurs, certaines personnes échouent à concilier la vie carcérale («faire son temps») et le maintien des liens familiaux. L'impossibilité de conserver concrètement, dans leurs relations familiales, leur statut et, subsidiairement, leur autorité peut inciter certains détenus à rompre volontairement tout lien et se protéger ainsi d'un possible abandon de leurs proches.

\section{Temps de dedans et temps de dehors}

Au-delà d'une tendance générale à la raréfaction des liens entre les personnes détenues et leurs proches au cours de l'incarcération, les expériences du temps debors et dedans (temps «libre» versus temps «sous contrôle») coïncident rarement, alors même que la solidarité s'exprime par le temps «donné» (notamment les visites). 


\section{«Tuer» le temps}

L'incarcération est souvent décrite par les personnes incarcérées comme un temps subi : "C'est comme un mal de dents où il n'y a rien à faire» (détenu). Il faut donc le prendre avec patience, car ce serait un «temps plus long que dehors», en raison de sa monotonie. Les jours passent et se ressemblent jusqu'à l'indistinction. En effet, le séquençage du temps est invariable, avec un emploi du temps (réveil, promenade, repas) immuable ${ }^{5}$ marqué, jour et nuit, par les rondes des surveillants.

La monotonie qui caractérise le temps en prison est décrite par les détenus comme s'accentuant au cours de la détention. Ils comptent le temps passé dans l'établissement en jours, puis en semaines, en mois, puis en années... Au début de l'incarcération, avant que la peine ne soit définitive, les échéances judiciaires (l'instruction, le jugement, les recours) sont des marqueurs importants du temps. Elles continuent à l'être, lorsque la peine devient définitive, pour la majorité des personnes incarcérées, car leur sortie approche ${ }^{6}$. Par contre, pour les détenus condamnés aux plus longues peines et affectés dans les établissements pour peine, où les libérations peuvent être exceptionnelles, les échéances judiciaires sont lointaines et les marqueurs du temps mobilisés ont un rapport avec l'établissement carcéral: les changements de cellule et d'établissement - qui peuvent être extrêmement rares -, parfois les changements de régime, mais aussi les événements mémorables de l'établissement (mouvement de protestation, suicide d'un codétenu ou arrivée d'un nouveau directeur par exemple) et les arrivées et les départs des codétenus.

La rigidité des temps institutionnels (heure des repas, des promenades, des douches, des visites, etc.) se traduit, pour les personnes détenues, par leur faible autonomie dans l'organisation de leur temps. Celui-ci est à la merci des temps institutionnels (de la prison et des instances judiciaires notamment) et à la disposition des surveillants, des travailleurs sociaux, mais aussi des proches. L'incarcération est décrite comme l'expérience d'un temps subi: c'est là l'une des caractéristiques essentielles des institutions totales décrites par Goffman (1968, p. 45).

5. Cette affirmation doit néanmoins être nuancée car, selon l'établissement d'affectation et leur situation pénale, les personnes détenues ont souvent, selon les jours de la semaine, des accès différenciés au travail, aux douches, aux activités socioculturelles et aux parloirs, aux cantines, à la possibilité de rencontrer un membre du personnel du Service pénitentiaire d'insertion et de probation (SPIP) ou un avocat notamment.

6. En 2016, la moitié des peines prononcées comportant une partie ferme d'emprisonnement étaient des peines inférieures ou égales à six mois d'incarcération (Löwenbrück, 2017). 
Or, dans les maisons d'arrêt comme dans les établissements pour peine, les détenus disent souvent qu'il faut «garder la tête dehors» et apprendre à «faire passer» le temps, car subir ce temps serait véritablement purger sa peine. Il faut donc s'occuper, notamment grâce au sport, au travail ou aux études (parfois des matières qui ne s'enseignent plus ailleurs ou des métiers pour lesquels il y a peu de perspectives d'emploi à l'extérieur). Le registre de l'occupation envahit toutes les dimensions de la vie carcérale - jusqu'à la masturbation. Cette volonté de s'occuper rejoint les préoccupations de l'Administration pénitentiaire (AP) et des membres du personnel, souvent davantage intéressés par l'aspect occupationnel des activités proposées en détention que par leur valeur propre ou par leur utilité pour la réinsertion des personnes détenues. À ce titre, le chercheur peut être instrumentalisé par les surveillants qui peuvent considérer l'entretien sociologique comme un moyen de «faire passer le temps» aux détenus.

Parce qu'il est synonyme de «mort lente» (pour reprendre une expression fréquemment utilisée pour évoquer les peines les plus longues) et d'ennui, le temps devient tangible et les détenus condamnés aux plus longues peines disent souvent qu'il faut le «combattre», le «tuer». Ces détenus peuvent avoir envie - quel qu'en soit le prix ultérieur à payer - de créer l'événement ou l'incident (bagarre, mutinerie) pour tuer l'ennui et justement marquer le temps. Relativement à ce temps décrit comme «sous contrôle», les occupations constituent une forme d'autonomie. Certains détenus indiquent même n'avoir «plus» le temps - sans être dupes parfois du paradoxe d'être si «occupés» quand la prison est ordinairement pensée comme un lieu d'ennui ou d'oisiveté (comme l'évoque ironiquement, par exemple, le surnom de «Poissyplage» donné à la maison centrale de Poissy).

\section{Le temps des autres}

On dit, dedans, «faire son temps». C'est d'ailleurs un aspect important des sociabilités carcérales que de s'enquérir de «quand» les codétenus sont arrivés et de «quand» ils sortiront - presque autant que des raisons pour lesquelles ils sont incarcérés. Les sociabilités entre proches de détenus, notamment lors des visites, sont également marquées par la question du temps: que l'on demande depuis quand on vient au parloir ou la date (probable) de sortie de la personne visitée, on s'enquière toujours de la durée de l'expérience passée et du temps qui reste à endurer. 
Le temps des proches est souvent assimilé à une attente, à commencer parce que dans le parler des prisons, une personne détenue qui est soutenue par ses proches est «attendue». Questionnés sur leurs liens avec la personne détenue, les proches évoquent souvent le temps qu'ils lui consacrent - un temps qui est "pris» par la prison, parce qu'au temps des visites s'ajoute celui passé dans les transports et dans les contrôles auxquels ils doivent se soumettre, mais aussi en démarches diverses (rencontre avec l'avocat, l'assistant social, etc.). Et ce temps dont ils soulignent fréquemment qu'ils le «donnent», c'est aussi celui qu'ils perdent (pour le travail, pour les loisirs, les sorties ou pour leurs amis).

Le temps est, entre dedans et dehors, source d'incompréhensions. Les proches se décrivent souvent comme hyperactifs et obligés à la mobilité (Touraut, 2009) et peuvent penser que les détenus sont eux inoccupés - qu'ils « ont leur temps». Or les personnes incarcérées, qui font l'expérience d'un temps «sous contrôle», opposent généralement celui-ci à un temps dehors qui serait «libre» car maîtrisable. Pourtant, les proches évoquent fréquemment le poids que font peser les rythmes institutionnels (horaires des visites, des appels téléphoniques, etc.) sur leur propre vie, mais aussi l'emprise du temps carcéral sur leurs activités: les conjointes en particulier décrivent leurs semaines, voire leurs journées comme rythmées par le travail domestique (entretien du linge, tâches administratives, etc.) et émotionnel (visites, courriers, etc.) qu'elles fournissent aux prisonniers.

La rupture entre les temps de dehors et de dedans se nourrit souvent d'un passé commun qui devient irréel. La force des expériences vécues en prison, comme celle de l'affrontement du système pénal, relègue irrémédiablement certains événements de la vie antérieure au second plan. Beaucoup de détenus, comme des proches, évoquent l'impression, avec le temps, de devenir étrangers les uns aux autres et d'avoir moins de choses à se raconter. Cela se traduit par la tendance, observée dedans comme dehors, de rédiger, à la longue, des lettres stéréotypées et de tenir, au parloir, des propos convenus, et finalement de se comporter comme on le fait d'ordinaire avec une personne gravement malade.

Entre dehors et dedans, c'est un temps qui sépare. Plusieurs détenus évoquent la souffrance d'être absents des moments traditionnels de réjouissance, comme les fêtes et les célébrations (les «temps forts») du groupe. Chaque absence signale davantage son «absence aux autres», alors que, simultanément, les moments de debors contribuent à baliser le temps de dedans («C'est mon deuxième Noël en prison»). Beaucoup 
de détenus évoquent ne plus se sentir concernés par certaines célébrations (l'anniversaire de naissance peut devenir moins significatif que celui de l'entrée dans l'établissement) et ne plus s'associer à certaines célébrations (anniversaires et fêtes religieuses notamment) ou le faire uniquement pour satisfaire leurs proches.

Les moments les plus durs, c'est l'été, on pense qu'on serait en vacances, à la plage, ou les samedis soir. [...] Et puis, le ramadan, c'est un peu difficile, alors j'appelle la famille tous les soirs. (N., 24 ans, condamné à 12 ans, incarcéré depuis 6 ans, en centre de détention)

Le temps de dedans ne coïncide pas avec celui de debors. Les personnes détenues peuvent se sentir en décalage ou en retard sur la vie dehors, notamment en raison de l'attente des lettres, des mandats et des nouvelles qui en viennent - et qui parfois n'arrivent pas. Plus généralement, Cohen et Taylor (1972, p. 99) évoquent un «effet accordéon»: les lettres, et plus encore les visites, interrompent la séparation par des condensés de présence de l'autre, des moments où il faudrait tout rattraper. Mais la rencontre des temporalités de dedans et de dehors ne se fait pas sans accrocs: le temps du parloir ${ }^{7}$ est celui de la frustration, puisqu'on est «déjà-après»: ce qui suit le parloir, c'est l'attente du prochain. Combien de fois entend-on au parloir: «Tu reviens quand?» Ces remarques doivent toutefois être prises avec prudence en raison de l'ancienneté de mes données. En effet, Cunha (2012) signale que ses observations, faites à la fin des années 1990 (Cunha, 1997), sur le temps dans une prison du Portugal et la manière dont il est rythmé par les visites, ne sont plus valides. Elle ne donne pas les raisons de cette évolution. Dans le contexte français, l'accès des personnes détenues à des téléphones (introduits illégalement) et donc aux réseaux sociaux ${ }^{8}$ atténue certainement le sentiment de décalage ou de retard avec l'extérieur d'une partie des personnes détenues.

\section{Donner du sens au temps et à la peine}

\section{L'avant-sortie}

Entre dehors et dedans, la sortie cristallise les façons diverses dont le temps de l'incarcération est vécu. La plupart des personnes détenues ne

7. Pour une exploration du parloir sous l'angle de la confrontation des temporalités entre dedans et dehors, voir Ricordeau, 2012.

8. Même si mes données ne l'ignorent pas, elles sont loin de prendre parfaitement la mesure du phénomène. 
savent pas exactement quand elles sortiront de prison, soit que leur peine n'a pas été prononcée, soit qu'elles espèrent obtenir une libération conditionnelle. Mais leurs proches en savent parfois encore moins en la matière. En effet, beaucoup de détenus rapportent ne pas dire précisément leur date de sortie à leurs proches (comme d'ailleurs les faits qui leur sont reprochés), notamment pour se protéger d'une pensée faite d'incertitudes et d'éventuels reproches. Certains rapportent même mentir, à l'instar d'un interviewé qui disait à ses proches chaque année, depuis plus d'une décennie, sortir «l'an prochain».

La projection de la sortie produit un processus d'acculturation (être dedans, mais déjà dehors). Cela explique notamment que les retours de permission soient, avec le temps, de plus en plus difficiles: chacune partage davantage le détenu entre dedans et dehors. Dans la perspective de ce changement de références, certains, dans les mois précédant la sortie, détruisent ce qui est associé à leur détention (les lettres reçues, les tatouages, etc.). Du reste, traditionnellement, les sortants distribuent leurs affaires («toto», radio, etc.) à leurs codétenus et codétenues. Plus la peine est longue, plus la sortie de prison est associée à des peurs, qui peuvent amener à «s'installer» en détention - un phénomène connu dans les hôpitaux psychiatriques sous le terme d'«asilisation» et plus généralement d'«installation» (Goffman, 1968, p. 107-108). Parce qu'il est monotone, voire immobile, le temps en dedans est sécurisant. Bénéficier de permissions de sortir n'atténue parfois pas l'angoisse de sortir, car elles entérinent parfois l'impression d'être devenu comme étranger au monde extérieur. Ce phénomène se nourrit du sentiment de beaucoup de personnes, incarcérées depuis de nombreuses années, de ne plus être «de leur temps», sentiment qui s'éprouve notamment devant les nouveaux téléphones portables, voitures, etc., et les habitudes langagières des «gens de dehors».

Comme la peine n'est pas d'une durée limitée, mais s'avère plutôt une interruption, une discontinuité par rapport au passé et au futur (Cunha, 1997, p. 61), la sortie suscite aussi des craintes chez les proches de détenus: peur d'être devenus des étrangers les uns aux autres, peur de ne plus savoir vivre ensemble, etc. L'incarcération d'un proche, même si elle se traduit par de nombreuses tâches domestiques, permet aussi de se préserver de certaines. La sortie peut donc être évoquée, par les proches (et singulièrement les compagnes), sous l'angle des aspects pratiques de la cohabitation, mais aussi de la charge (en termes de travail domestique et émotionnel) qu'elle peut représenter. 
Le pire, c'est qu'ils sont pareils dedans qu'ils étaient dehors. Et ouais, ça les rend pas moins cons d'être au placard, ce serait même plutôt le contraire... Alors il va falloir se le fader quand il va ressortir! (B., épouse de détenu)

Des fois, avec mes autres frères et sœurs, et même avec la frangine de mon mari, on se dit que dès qu'ils seront à nouveau ici, c'est pas d'être allé en taule qui les empêchera de nous prendre la tête! On rigole, mais c'est vrai... Lautre fois, en délirant, on se disait qu'à la moindre prise de tête, on demande à l'AP [l'Administration pénitentiaire] de nous les reprendre. Parce qu'en vrai, ils sont pas tous les jours cool... (C., sœur et épouse de détenu)

\section{Temps perdu et temps rationalisé}

Les détenus et leurs proches conçoivent rarement l'incarcération comme une «simple» parenthèse. En effet, comme le dit un dicton de prison ("On ne sort pas de prison, on sort avec la prison»), la vie dehors demeure sous l'emprise de dedans, à commencer par le rythme carcéral (lever, repas et coucher notamment) que continue de suivre le sortant. Mais l'incarcération a aussi des effets physiologiques, sur les sens et la sexualité (en particulier pour les personnes qui purgent de longues peines). Pour les proches, ces effets peuvent demander un travail de «maternage».

En plus, il paraît qu'on les récupère fainéants! Tu vois le cadeau! Il paraît qu'il y a des conséquences... Sur la vue, pour conduire, traverser la rue... Bah, faut être aux petits soins avec eux quand ils sortent, ils sont déboussolés... ça va pas être de la tarte! (S., compagne de détenu)

Le temps est certes expérimenté et rationalisé différemment selon la durée de la peine et ses conséquences (sociales et familiales), mais aussi le type d'établissement dans lequel la personne a été affectée et le type de régime subi. À ceux qui jugent leur peine injuste, disproportionnée, inutile (le temps «n'a jamais fait revenir» une victime) ou sans rapport avec leurs actes (notamment les auteurs de délits ou crimes à caractère sexuel), s'opposent ceux (les «voyous») pour lesquels la peine est la contrepartie d'un mode de vie choisi et ceux dont le sentiment de culpabilité n'est extinguible qu'avec l'assurance de «payer» pour l'acte commis (auteurs de crimes dits «passionnels» notamment).

L'incarcération peut être conçue comme l'occasion d'échapper à un sort inéluctable (notamment pour les personnes ayant un parcours toxicomaniaque) ou à son sort («J'ai eu de la chance d'aller au placard, 
je suis devenue quelqu'un d'autre, quelqu'un de mieux»). La valorisation de l'expérience carcérale, en tant qu'épreuve surmontée, mais aussi par la rencontre de personnes différentes de celles du milieu social d'origine (par exemple des bénévoles ou des enseignants), peut aussi se décliner en termes de qualités morales acquises (la tolérance, la compréhension ou la compassion). Il ne faut néanmoins pas être dupe de la mise en forme du passé que la recherche par interview suscite - qui encourage à trouver du sens au temps carcéral, mais aussi à présenter à la sociologue un discours qui pourrait être tenu devant une commission d'application des peines.

La considération de l'incarcération comme du «temps perdu» ou un «temps mort» peut avoir été combattue par une rationalisation du temps passé en détention, par les études ou le travail notamment. Ceux-ci peuvent permettre de trouver, à la libération, un travail dont l'honnêteté est opposée aux «conneries» antérieures. L'incarcération peut également marquer véritablement le début d'une nouvelle vie en raison des décisions prises pendant la peine, résultat d'une réflexion sur soi-même.

Mon mari, il a neuf diplômes. Il les a tous passés en prison. C'est un travailleur... Il m'a fait plein de cadeaux de choses qu'il fait à l'intérieur. Il dessine aussi... Quand il sortira, y a pas de souci, il trouvera du travail, même si c'est pas très bien payé. Ah ça, il le dit toujours: «Les conneries, c'est fini!» Vous imaginez, il a été condamné à perpétuité quand même... Alors il a vraiment envie de s'en sortir... (S., compagne de détenu)

\section{Au-delà de la peine}

Si l'incarcération n'est pas considérée de manière routinière, les détenus expriment souvent le désir de «commencer leur vie» à leur sortie : une vie comme "celle de tout le monde», avec une famille et un travail comme gages de normalité. Mais l'incarcération peut avoir profondément changé les relations avec les proches et ceux-ci peuvent aussi avoir changé en raison de l'épreuve qu'ils ont traversée.

À ma sortie, je sais que je vais retrouver ma femme beaucoup plus forte. L'autre jour, elle m’a dit: «Je suis blindée.» Vous savez, après tout ce qu'elle a subi, et ce qu'elle continue de subir, à part la mort, il n'y a plus grandchose de pire! Surtout, ma femme, mais aussi mon fils, ils ont changé parce qu'ils ont tenu le coup. (G., 44 ans, condamné à 22 ans, incarcéré depuis 4 ans, en centre de détention) 
On ira s'installer à la montagne. Ça fait six ans qu'on en rêve... Mon mari, il dit toujours que le béton, c'est pas fait pour lui... Après quinze ans de prison, il ne veut plus en voir! (S., compagne de détenu)

La sortie est souvent comparée, par les futurs ex-détenus à une «renaissance», où les «compteurs sont remis à zéro». Pourtant la sortie constitue très rarement un rite de réintégration qui compenserait, sur le plan symbolique, la «cérémonie de dégradation» qu'est l'entrée en prison (Garfinkel, 1956, p. 420-424). Mais la sortie de prison fait aussi surgir le spectre d'y retourner, surtout pour les personnes qui ont effectué plusieurs peines. Le souci des sortants (combien de temps on va savoir «rester dehors») fait écho à celui des proches, qui évoquent parfois l'incarcération et la sortie comme des routines, avec leur lot de contraintes. À l'inverse, certains proches, en particulier des compagnes (et notamment celles qui ont rencontré leur compagnon lorsque celui-ci était déjà incarcéré), considèrent que leur rôle inclut d'œuvrer à la réinsertion, voire de surveiller, le sortant de prison.

Mon mari, à la première connerie, c'est moi qui le dénonce. Ben ouais! Parce qu'il y a des femmes, moi je les qualifie de «femmes de voyou». Moi, c'est pas ma mentalité. Et puis, c'est pas leur rendre service. La juge [de l'application des peines], elle le sait de toute façon. Moi, je suis fille de gendarme, alors comme je lui ai écrit: «Je me porte garante $d^{\prime} E^{\star \star \star}$. D'ailleurs, s'il est libéré, je vous donnerai des nouvelles tous les ans.» (M., épouse de détenu)

\section{Conclusion}

«Savoir quand on sort» a longtemps caractérisé la prison, a contrario de l'hôpital psychiatrique. Cela s'avère de moins en moins exact avec l'allongement des peines prononcées, la difficulté d'obtention des aménagements de peine, la longueur des périodes de détention préventive et la rétention de sûreté. Par ailleurs, malgré les recommandations en termes d'individualisation des peines et de préparation à la sortie des personnes détenues, celles-ci associent souvent l'incarcération à un «temps perdu». En suivant toujours le sens trigonométrique, c'est d'ailleurs comme à rebours du temps que semble aller la marche des détenus dans les cours de promenade.

Malgré la faible autonomie dont ils disposent en détention, les stratégies que les détenus peuvent mettre en place pour rationaliser le temps passé entre les murs sont nombreuses. Leurs proches, même s'ils 
peuvent toujours se dérober aux injonctions à la solidarité conjugale et familiale, subissent aussi l'emprise des temporalités carcérales et des rythmes institutionnels. Si le temps passé dedans a soulevé de nombreux débats visant à rendre ce temps utile pour l'individu et la société, le temps dehors que prend, pour leurs proches, la solidarité avec les personnes détenues est rarement discuté. Que ce temps-là soit en grande partie le temps des femmes et consacré à des tâches typiquement féminines (travail domestique et émotionnel notamment) est sans doute un indice pour comprendre le désintérêt qui a jusqu'à présent entouré cette question - et la nécessité politique de s'en emparer.

\section{Références}

Adams, K. (1992). Adjusting to prison life. Crime and Justice, 16, 275-359.

Braman, D. (2007). Doing time on the outside: Incarceration and family life in urban America. Ann Arbor, MI: University of Michigan Press.

Cassan, F., Toulemon, L. et Kensey, A. (2002). L'histoire familiale des hommes détenus. INSEE Première, (706).

Chantraine, G. (2003). Prison, désaffiliation, stigmates. L'engrenage carcéral de l'«inutile au monde» contemporain. Déviance et Société, 4(27), 363-387.

Clemmer, D. (1940). The prison community. New York, NY: Rinehart \& Winston.

Cohen, S. et Taylor, L. (1972). Psychological survival: The experience of long term Imprisonment. Middlesex, Royaume-Uni: Penguin Books.

Comfort, M. (2008). Doing time together: Love and family in the shadow of the prison. Chicago, IL: University of Chicago Press.

Cunha, M. I. (1997). Le temps suspendu. Rythmes et durée d'une prison portugaise. Terrain, 29, 59-68.

Cunha, M. I. (2012). Une prison à l'épreuve du temps: temporalités carcérales d'hier et d'aujourd'hui. Dans S. Humbert, N. Derasse et J.-P. Royer (dir.), La prison: du temps passé au temps dépassé (p. 143-153). Paris, France: L'Harmattan,

Direction de l'Administration pénitentiaire. (2018). Statistique mensuelle des personnes écrouées et détenues en France. Situation au 1er juillet 2018. Paris, France: Ministère de la Justice.

Fabiani, J.-L. et Soldini, F. (1995). Lire en prison. Paris, France: BPI.

Garabedian, P. G. (1963). Social roles and processes of socialization in the prison community. Social Problems, 11(1), 139-152.

Garfinkel, H. (1956). Conditions of successful degradation ceremonies. American Journal of Sociology, 61(5), 420-424.

Goffman, E. (1968). Asiles. Étude sur la condition sociale des malades mentaux et autres reclus. Paris, France: Minuit.

Irwin, J. et Cressey, D. R. (1962). Thieves, convicts and the inmate culture. Social Problems, 10(2), 142-155. 
Leal, W. C. et Mond, D. (2001). From my prison cell: Time and space in prison in Columbia, an ethnographic approach. Latin American Perspectives, 28(1), 149-164.

Löwenbrück, M. (2017). L'évolution des peines d'emprisonnement de 2004 à 2016. Infostat Justice, (156). Paris, France: Ministère de la Justice. Repéré à http://www.justice.gouv.fr/art_pix/stat_Infostat_156.pdf

Marchetti, A. M. (2001). Perpétuités. Le temps infini des longues peines. Paris, France: Plon.

Middlemass, K. M. et Smiley, C. J. (2016). Doing a bid. The construction of time as punishment. The Prison Journal, 96(6), 793-813.

Montandon, C. et Crettaz, B. (1981). Paroles de gardiens, paroles de détenus. Genève, Suisse: Masson.

Moran, D. (2012). "Doing time" in carceral space: Timespace and carceral geography. Geografiska Annaler: Series B, Human Geograpby, 94(4), 305-316.

Muth W. et Walker, G. (2013). Looking up: The temporal horizons of a father in prison. Fathering: A Journal of Theory, Research, and Practice About Men as Fathers, 11(3), 292-305.

Pauchet, C. (1984). Le temps en milieu carcéral (temps institutionnel et temps vécu). Revue pénitentiaire et de droit pénal, 106(3), 296-302.

Ricordeau, G. (2008). Les détenus et leurs proches. Solidarités et sentiments à l'ombre des murs. Paris, France: Autrement.

Ricordeau, G. (2012). Entre dehors et dedans: Les parloirs des prisons, Politix, 25(97), 101-123.

Touraut, C. (2009), Entre détenu figé et proches en mouvement. «L'expérience carcérale élargie» : une épreuve de mobilité. Recherches familiales, 1(6), 81-88.

Touraut, C. (2012). La famille à l'épreuve de la prison. Paris, France: Presses universitaires de France.

Wheeler, S. (1961). Socialization in correctional communities. American Sociological Review, 26(5), 697-712.

\title{
"Doing time" and "waiting": Carceral rhythms and the experience of time inside and outside prison
}

\begin{abstract}
This article draws on interview-based research among relatives of prisoners and inmates in France. It explores the different ways in which time is experienced inside and outside prison during different phases of incarceration and discusses how this experience relates to other social rhythms, particularly the way in which inmates are encouraged to "make sense" of their sentence or "do their time", while relatives of prisoners are expected only to wait. I look at the different ways time is experienced (inside and outside prison), which vary depending upon sentence length and time already served. I then describe various forms of incompatibility between time as experienced inside and outside prison and discuss how this affects family relationships. Finally, I analyze how inmates, ex-inmates, and their relatives develop rationalizations to deal with a period of time initially seen as wasted and how these strategies are influenced by sentence length and type of prison.
\end{abstract}

KEYWORDS - Prison, inmates, visit, time, relatives. 


\section{"Cumplir su pena" y "esperar" : temporalidades carcelarias y el tiempo vivido adentro y afuera}

RESUMEN - Basado en una investigación de campo realizada en Francia con personas detenidas y con familiares de prisioneros, este artículo aborda las diversas formas en las que la pena es vivida, adentro y afuera, durante las diferentes fases del encarcelamiento y cómo estas se confrontan con otros ritmos sociales, en particular con el mandato dirigido a los prisioneros de "darle un sentido a su pena" (o "cumplir su condena"), mientras que los familiares son asignados en una posición de espera. En primer lugar, la autora describe las diferentes experiencias del tiempo de la pena (adentro y afuera) que varian según la duración de la pena y el tiempo ya pagado. Describe después diversas formas de discordancia entre el tiempo vivido adentro y afuera y la manera en la que se traducen en las relaciones y en las solidaridades familiares. Finalmente, la autora analiza de qué manera los prisioneros, los ex prisioneros y sus familiares racionalizan un tiempo inicialmente pensado como "perdido" y cómo sus estrategias están influenciadas por la duración de la pena y por el tipo de cárcel en la que se paga la pena.

PALABRAS CLAVE • Cárcel, detenidos, detenidas, visita, tiempo, familiares. 